\title{
Lunge und Gelenke in Gefahr
}

Sie sind keine Seltenheit und sie sind „sehr bunt“: Lungenbeteiligungen bei rheumatoiden Erkrankungen, allen voran die RA-assoziierte interstitielle Lungenkrankheit, haben einen erheblichen Einfluss auf die Prognosen der Patienten. Sie zu übersehen ist folgenschwer. Deshalb: Genau hinsehen und handeln!

Eine interstitielle Lungenkrankheit (ILD; Interstitial Lung Disaease) findet sich bei genauem Hinschauen begleitend bei der Mehrzahl der Rheumaerkrankungen, etwa im Rahmen einer systemischen Sklerose (SSc), einer Dermatomyositis oder einer rheumatoiden Arthritis (RA), bei der auch die Atemwege im pleuralen und vaskulären Bereich betroffen sein können, erläuterte PD Dr. Francesco Bonella, Universitätsmedizin Essen.

An eine Lungenbeteiligung denken Oft bleiben diese ILDs unerkannt. Die Veränderungen der Lunge lassen sich im CT bei $60 \%$ der Patienten erkennen, aber nur $5 \%$ der RA-Patienten zeigen Symptome. Bei systemischer Sklerose zeigen $75 \%$ in der Autopsie eine Lungenbeteiligung, aber nur $40 \%$ waren symptomatisch. Heißt also: Man muss bei einer rheumatoiden Erkrankung an eine Lungenbeteiligung denken.

) Eine ILD findet sich bei genauem Hinschauen begleitend bei der Mehrzahl der Rheumaerkrankungen

Umgekehrt kann eine ILD aber auch die Erstmanifestation einer rheumatoiden Erkrankung sein. So entwickeln 10 bis $15 \%$ der RA-Patienten im Vorfeld eine ILD, weniger als bei einer Myositis mit $30 \%$ und ähnlich viele wie bei einer SSc mit $15 \%$. Dass das Thema relevant ist, zeigen die Daten einer topaktuellen Studie, die eine steigende Prävalenz der RA-ILD zeigt, allerdings mit sinkender Mortalität.

Wie wichtig es ist, die ILD so früh wie möglich zu erkennen, zeigt eine multizentrische spanische Studie mit $106 \mathrm{~Pa}-$ tienten. Das Gesamtüberleben der Pa- tienten war abhängig vom Zeitpunkt der Diagnosestellung: Eine verspätete Diagnose ging mit einer schlechten Prognose einher, vor allem wenn die ILD erst nach einem oder zwei Jahren erkannt wurde.

\section{Prognostische Faktoren erkennen} Aus Sicht von Bonella gehört zum Management der Erkrankung die Identifikation prognostischer Faktoren. Ungünstig sind etwa der Schweregrad von Traktionsbronchiektasen, das Ausmaß der Lungenfibrose im CT und die Reduktion im DLco. Ein UIP-Muster bei RA reduziert das Überleben ähnlich einer idiopathischen Lungenfibrose (IPF). Eine Fibrose im HRCT > $20 \%$ gelte ebenfalls als prognostisch negativ, so Bonella.

Immunsuppression und Antifibrose Die Therapie stützt sich auf die klassischen sDMARDs, zum Beispiel Azathioprin, Methotrexat (MTX), Leflunomid und bei aggressivem Verlauf auch Cyclophosphamid, ebenso wie auf bDMARDS; darunter Tocilizumab, Rituximab und Abatacept. Unter Abatacept wurde eine Stabilisierung oder leichte Verbesserung von Vitalkapazität und DLco innerhalb von zwölf Monaten beobachtet, allerdings mit einem anschließenden Abfall der Lungenfunktion.

\section{》) Interstitielle Lungenkrankheiten} bleiben oft unerkannt

Für Rituximab gibt es Daten über einen günstigen prognostischen Effekt mit einem Überlebensvorteil. Noch für 2021 werden die Ergebnisse der RECITAL-Studie erwartet, die Rituximab mit Cyclophosphamid ver- gleicht. Bonella wies zudem explizit darauf hin, dass MTX vor der Entstehung einer ILD bei RA eher schützt. Dies zeigten Metaanalysen und retrospektive Studien inzwischen eindeutig. MTX sei nicht assoziiert mit einem erhöhten Risiko einer RA-ILD bei RA. Man muss keine Befürchtungen haben, eine MTX-Pneumonie sei etwas völlig anderes.

》) Eine ILD kann auch die Erstmanifestation einer rheumatischen Erkrankung sein

Eingesetzt in der Therapie der RA-ILD werden auch Antifibrotika - mit Erfolg. Sowohl für Nintedanib als auch für Pirfenidon gebe es signifikant positive Daten auch bei RA-ILD. Ziel der Therapie müsse es vor allem sein, akute Exazerbationen zu verhindern, denn eine akute Exazerbation bei RA-ILD gehe mit einer schlechten Prognose einher.

Hinweis des Verlags. Der Verlag bleibt in Hinblick auf geografische Zuordnungen und Gebietsbezeichnungen in veröffentlichten Karten und Institutsadressen neutral.

rheuma plus $2021 \cdot 20: 258$

https://doi.org/10.1007/s12688-02100462-7

(c) Springer-Verlag GmbH Austria, ein Teil von Springer Nature 2021 